\section{Degradabilidade da fibra do bagaço de cana-de-açúcar tratado por soluções alcalinas, pela técnica dos sacos de náilon "in situ"}

\author{
Fiber degradability of sugar cane bagasse treated by alkaline \\ solutions, through the "in situ" nylon bag technique
}

Sérgio Carlo l' ranco MOR(jULIS ', Laércio MEL(OTTI ${ }^{3}$, Carlos de Sousa LUCCI ", Oziel BIZUTTI ", Gilberto BRAUN +
CORRESPONDENCE TO Carlos de Sousa Lucci Departamento de Criação de Ruminantes e Alimentação Anima: Faculdade de Medicina Veterinária e Zootecnia da USP

Av. Duque de Caxias Norte, 225 Caixa Postal 23

13630-970 - Pirassununga - SP - Brasit

1 - Autônomo - São Paulo - SP 2 - Faculdade de Medicina Veterinária e Zootecnia da USP - SP 3 - Instituto de Zootecnia - Nova Odessa-SP

\title{
RESUMO
}

O bagaço de cana-de-açúcar (BCA) foi submetido a três tratamentos, sendo imerso em uma das seguintes soluções: (A) de $\mathrm{NaOH}$ ( $2 \%$ peso/volume), (B) de cinzas de madeira (30\% peso/volume) e (C) em água. E constituiu $60 \%$ da matéria seca das dietas, junto com $40 \%$ de concentrados (milho, grão de soja e farelo de algodão), fornecidas em ensaios de degradabilidade ruminal para 6 bovinos com fístulas ruminais. O delineamento foi o "change-over" (3x3), adotando-se a técnica de sacos de náilon "in situ". A degradabilidade da MS às $48 \mathrm{~h}$ foi $(p<0,05):(A)=75,14 \%,(B)=$ $34,20 \%,(C)=24,26 \%$. A degradabilidade da fração FDN do BCA foi sempre maior $(p<0,05)$ no tratamento $(A)$ do que nos demais; já a degradabilidade no tratamento $B$ foi maior $(p<0,05)$ do que no $C$, às 72 e $96 \mathrm{~h}$. A degradabilidade da FDN às 48 h de incubação foi: $(A)=74,01 \%,(B)=41,61 \%$ e $(C)=35,72 \%$. Concluiu-se que os tratamentos alcalinos (soda e cinzas de madeira) melhoram a degradabilidade ruminal do BCA, sendo a soda superior às cinzas.

UNITERMOS: Cana-de-açúcar; Bovinos; Digestibilidade.

\section{INTRODUÇĀO E REVISÃO DE LITERATURA}

$\mathrm{A}$ indústria sucroalcooleira nacional produz anualmente cerca de 50 milhões de toneladas de bagaço de cana-de-açúcar (BCA). Grande parte desta produção, em torno de $80 \%$, é utilizada nas próprias usinas e destilarias para gerar energia.

Esse subproduto tem um excedente de aproximadamente 10 milhões de toneladas, daí sua utilização no Brasil na alimentação animal estar sendo cada vez mais difundida nos últimos anos. O emprego deste alimento vem sendo feito "in natura" ou tratado por diversos processos; e, entre esses processos, a imersão do bagaço em soluções alcalinas deve ser mais bem estudado.

Tratamento alcalino dos subprodutos agrícolas ricos em fibra tem melhorado o seu valor nutritivo para ruminantes

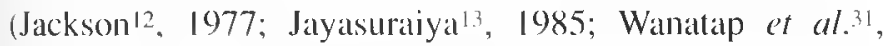
1986); as cinzas de madeira, estudadas por Bergner (1981) apud Owen of al.25 (1984); Adebowalel (1985); Nolte et al.22 (1987); Freitas; Patrika' (1989); Sudana29 (1990) e Ramõrez et al.27 (1991), também merecem destaque, pois contêm muitos componentes alcalinos.

O tratamento do BCA por $\mathrm{NaOH}$ foi avaliado por vários autores (Lamas et al..5, 1979); Orskov et al.23, 1980); Cabello et al. ${ }^{2}, 1981$; Reddy et al.28, 1981; Chicco et al. ${ }^{6}, 1983$;
Ibrahin; Pearcel1, 1983; Lavezzo et al. ${ }^{16}$, 1983; Molina et al.21, 1983; Vitti et al. ${ }^{30}, 1985$; Martin 18, 1988), tendo sido observados resultados positivos na maioria dos trabalhos.

O objetivo deste experimento foi o de avaliar a ação de tratamentos no bagaço de cana-de-açúcar por soluções alcalinas de soda cáustica ou de cinzas de madeira, comparados à testemunha (imersão em água), sobre a degradabilidade ruminal da matéria seca e da fibra em detergente neutro.

\section{MATERIAL E MÉTODO}

Para o ensaio de degradação ruminal com sacos de náilon "in situ", foram utilizados 6 bovinos fistulados com peso médio de $400 \mathrm{~kg}$, nass instalações da Faculdade de Medicina Veterinária e Zootecnia da Universidade de São Paulo, em Pirassununga, SP. Utilizou-se bagaço de cana-de-açúcar recém-moída, com aproximadamente $50 \%$ de MS, para compor três tratamentos (diferentes processamentos do BCA $): A=$ por solução de soda $(p H=11,5) ; B=$ por solução de cinzas $(\mathrm{pH}=11,0) ; \mathrm{C}=$ por água $(\mathrm{pH}=5,(0)$.

$\mathrm{O}$ bagaço foi imerso por 6 horas em soluções de $\mathrm{NaOH}$ a $2 \%$ p.v. (peso/volume), ou cinzas de madeira a $30 \%$ p.v., ou em água, na proporção de $100 \mathrm{~kg}$ de bagaço para 700 litros. posteriormente deixando-se escorrer por 18 horas o excesso 


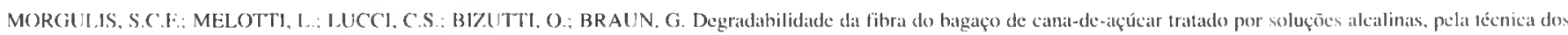

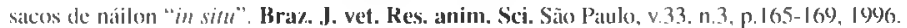

\section{Tabela 1}

Ingredientes usados nas rações dos diversos tratamentos, em porcentagem na matéria seca. Pirassununga SP, 1990.

\begin{tabular}{lccc}
\hline $\begin{array}{l}\text { Ingredientes } \\
(\%)\end{array}$ & $\begin{array}{c}\text { Soda } \\
(\mathrm{A})\end{array}$ & $\begin{array}{c}\text { Cinzas } \\
(\mathrm{B})\end{array}$ & $\begin{array}{c}\text { Água } \\
(\mathrm{C})\end{array}$ \\
\hline Bagaço MS (65 C) & 60,0 & 60,0 & 60,0 \\
Grão de milho moído & 16,4 & 16,4 & 16,4 \\
Soja grão moída & 10,4 & 10,4 & 10,4 \\
Farelo de algodão & 10,4 & 10,4 & 10,4 \\
Premix mineral & 1,8 & 1,8 & 1,8 \\
Premix vitamínico & 1,0 & 1,0 & 1,0 \\
\hline
\end{tabular}

de líquido antes da alimentação.

Os tratamentos compreenderam $60 \%$ da dieta com bagaço e os ingredientes que constituíram as rações constam da Tab. 1.

Durante todo o transcurso do período experimental, foram adicionados $25 \mathrm{ml}$ de uma solução aquosa de uréia $(48 \%)$ e sulfato de sódio $(8 \%)$, via fístula ruminal, uma hora após cada uma das duas refeições, com finalidade de aumentar o nível de nitrogênio não protéico das dietas.

O delineamento estatístico foi do tipo "change-over" (Cochran; $\operatorname{Cox}^{7}, 1957$ ) com 2 grupos de 3 animais cada. As médias foram comparadas pelo teste de Tuckey (Cochran; Cox 7,1957$)$.

Os sacos de náilon utilizados para incubação dos alimenlos no rúmen mediam $7 \times 17 \mathrm{~cm}$, com porosidade de cerca de 50 micra. Os pesos aproximados das amostras foram de $5 \mathrm{~g}$.

Três subperíodos experimentais de 28 dias cada un compreenderam: adaptação à dieta (2I dias) e coleta de amostras para a avaliação da degradação, $\mathrm{pH}$ e "furn over" ruminais ( 7 dias).

Os alimentos volumosos e concentrados foram misturados e ofertados em duas refeições iguais, às $8 \mathrm{~h}$ e às $16 \mathrm{~h}$. Amostras do bagaço de cana foram oblidas dos tratamentos com soluções (soda, cinzas, e água), posteriormente secas e moídas. Foram separados $2 \mathrm{~kg}$ destas para utilização nos sacos de náilon a serem incubados no rúmen.

A técnica utilizada para incubação do alimento no rúmen foi descrita por Orskov et al..$^{23}$ (1980), com algumas adaptações, constantes em Melotti et al. ${ }^{19}$ (1994). Os tempos de incubação ruminal utilizados foram: $0 ; 6 ; 12 ; 24 ; 48 ; 72$ e 96 horas (matéria seca (MS) e fibra em detergente neutro $(\mathrm{FDN}))$.

A obtenção da variável Taxa de Degradação foi efeluada utilizando-se a curva de desaparecimento das frações analisadas, segundo o modelo matemático proposio por Orskov; McDonald 24 (1979).

Após a retirada do último saco de náilon, na prova de degradabilidade, introduziram-se através da fístula ruminal, uma hora antes da primeira refeição, 300 g do marcador de fase líquida polietileno-glicol (PEG peso molecular 4000), conforme técnica descrita por Hyden ${ }^{10}$ (1986). Amostras de líquido ruminal para determinação do "turn over" ruminal, foram colhidas às ()h (antes da introdução do PEG); Ih; 3h; 6h; $9 \mathrm{~h} ; 12 \mathrm{~h}$ e $24 \mathrm{~h}$.

\section{RESULTADOS E DISCUSSÄO}

A análise químico-bromatológica do BCA, nos diferentes tratamentos, apresentou os resultados constantes na Tab. 2.

Houve um decréscimo do porcentual da FDN da ordem de 20 unidades para o subproduto tratado $\mathrm{com} \mathrm{NaOH}$ (A), e de 10 para o tratado com cinzas de madeira (B), relativamente ao tratado com água (C). Já o decréscimo porcentual da FDA foi muito menor, de 4 e de 5 unidades para soda e cinzas respectivamente, em relação ao testemunha, sugerindo que a ação de soda e de cinzas ocorreu com mais intensidade na fração hemicelulose do BCA.

A fração FDN do BCA tratado com $\mathrm{NaOH}$ apresentou redução da ordem de $21,0 \%$, encuanto a fração FDA loi de $4,1 \% \mathrm{em}$ relação ao produto não tratado. Esses resultados

Tabela 2

Resultados de análise do BCA e das rações experimentais nos diferentes tratamentos, em porcentagens na matéria seca. Pirassununga - SP, 1993.

\begin{tabular}{l|rrr|rrr}
\hline \multirow{2}{*}{ NUTRIENTES } & \multicolumn{6}{|c}{ TRATAMENTOS } \\
\cline { 2 - 7 } & \multicolumn{3}{|c|}{ Bagaço de cana (BCA) } & \multicolumn{3}{c}{ Rações experimentais } \\
\cline { 2 - 7 } FDN & \multicolumn{1}{|c|}{ Soda } & Cinzas & Água & A Soda & B Cinzas & C Água \\
\cline { 2 - 7 } & 73,87 & 84,18 & 94,86 & 70,00 & 71,90 & 70,32 \\
FDA & 55,17 & 53,81 & 59,23 & -- & -- & - \\
MM & 15,05 & 10,18 & 1,26 & 9,37 & 6,74 & 4,05 \\
PB & -- & -- & - & 11,00 & 12,45 & 12,20 \\
\hline
\end{tabular}


Tabela 3

Resultados do desaparecimento da matéria seca do $\mathrm{BCA}$, em diferentes tempos de incubação ruminal, e os respectivos coeficientes de variação. Pirassununga - SP, 1990.

\begin{tabular}{l|c|c|c|r}
\hline \multirow{2}{*}{$\begin{array}{l}\text { Tempos de } \\
\text { incubação }\end{array}$} & \multicolumn{4}{|c}{ Porcentual de desaparecimento da MS } \\
\cline { 2 - 5 } & Soda & Cinzas & \multicolumn{1}{c}{ Água } & \multicolumn{1}{c}{ C.V.\% } \\
\hline $0 \mathrm{~h}$ & 25,25 & 13,45 & 8,56 & - \\
$6 \mathrm{~h}$ & $33,35 \mathrm{a}$ & $11,34 \mathrm{~b}$ & $7,26 \mathrm{c}$ & 11,17 \\
$12 \mathrm{~h}$ & $45,68 \mathrm{a}$ & $16,43 \mathrm{~b}$ & $9,96 \mathrm{c}$ & 9,40 \\
$24 \mathrm{~h}$ & $59,98 \mathrm{a}$ & $27,23 \mathrm{~b}$ & $16,58 \mathrm{c}$ & 12,49 \\
$48 \mathrm{~h}$ & $75,14 \mathrm{a}$ & $34,20 \mathrm{~b}$ & $24,26 \mathrm{c}$ & 8,47 \\
$72 \mathrm{~h}$ & $83,29 \mathrm{a}$ & $37,88 \mathrm{~b}$ & $27,96 \mathrm{c}$ & 8,92 \\
$96 \mathrm{~h}$ & $86,21 \mathrm{a}$ & $41,74 \mathrm{~b}$ & $31,85 \mathrm{c}$ & 7,42 \\
\hline
\end{tabular}

abc - Letras diferentes, na mesma linha, indicam diferenças $(p<0,05)$ pelo teste de Tuckey.

\section{Tabela 4}

Resultados do desaparecimento de FDN do BCA, em diferentes tempos de incubação ruminal, e os respectivos coeficientes de variação. Pirassununga - SP, 1990.

\begin{tabular}{l|c|c|c|r}
\hline \multirow{2}{*}{$\begin{array}{l}\text { Tempos de } \\
\text { incubaçāo }\end{array}$} & \multicolumn{4}{|c}{ Porcentual de desaparecimento da FDN } \\
\cline { 2 - 5 } & Soda & Cinzas & Água & \multicolumn{1}{c}{ C.V.\% } \\
\hline $0 \mathrm{~h}$ & 21,40 & 17,92 & 16,26 & ---- \\
$6 \mathrm{~h}$ & $28,97 \mathrm{a}$ & $16,09 \mathrm{~b}$ & $16,26 \mathrm{~b}$ & 11,17 \\
$12 \mathrm{~h}$ & $39,44 \mathrm{a}$ & $21,40 \mathrm{~b}$ & $18,96 \mathrm{~b}$ & 9,40 \\
$24 \mathrm{~h}$ & $57,84 \mathrm{a}$ & $31,71 \mathrm{~b}$ & $27,57 \mathrm{~b}$ & 12,49 \\
$48 \mathrm{~h}$ & $74,01 \mathrm{a}$ & $41,61 \mathrm{~b}$ & $35,72 \mathrm{~b}$ & 8,47 \\
$72 \mathrm{~h}$ & $82,94 \mathrm{a}$ & $45,23 \mathrm{~b}$ & $39,03 \mathrm{c}$ & 8,92 \\
$96 \mathrm{~h}$ & $86,28 \mathrm{a}$ & $46,03 \mathrm{~b}$ & $40,96 \mathrm{c}$ & 7,42 \\
\hline
\end{tabular}

abc - Letras diferentes, na mesma linha, indicam diferenças $(p<0,05)$ pelo teste de Tuckey.

foram similares aos obtidos por Lamas et al.15 (1979), Ibrahin; Pearcell (1983) e Molina et al.21 (1983) que também utilizaram o $\mathrm{NaOH}$ com agente alcalinizante.

O valor de FDN observado no BCA tratado por cinzas de madeira, de $84.18 \%$ (Tab. 2), foi próximo ao encontrado por Molinat et al. ${ }^{21}$ (1983), 86,00\% com 4\% de $\mathrm{NaOH}$, e Ibrahin; Pearcell (1983), 84,5\% com 3\% de $\mathrm{NaOH}$.

É importante notar que, quando comparamos a concentração da FDN, nos tratamentos soda e cinzas o decréscimo obtido por Ibrahin; Pearcell (1983) (para 6\% de $\mathrm{NaOH}$ ) foi de 9.9 pontos e por Molina et al.21 (1983), de 8,5, próximos ao do presente trabalho, em que foi achado 10,31 entre os releridos tralamentos.

É interessante ressaltar que a literatura cita diferentes teores de NaOH utilizados (Lamas et al. ${ }^{15}$, 1979; Cabello et al.2. 1981: Ibrahin: Pearcel1, 1983; Molina et al.21, 1983) e que níveis maiores de concentração de $\mathrm{NaOH}$ nos tratamentos provocal perda crescente de FDN no produto tratado, aumento na Matéria Mineral (MM) e poucas alterações na
FDA, em relação ao produto não tratado.

O teor da MM evidencia a alteração provocalda no produto pelos tratamentos: com soda (A) e com cinzas (B), o que parece indicar o incremento no teor de minerais. Os resultados obtidos foram próximos aos de Ibrahin; Pearcell (1983), e o conteúdo mineral residual do $\mathrm{BCA}$ tratado e o $\mathrm{BCA}$ não tratado foi de 13,8 unidades, para o BCA tratado por soda (A).

A Tab. 3 apresenta os resultados do desaparecimento da MS $(65$ C) do BCA nos diferentes tempos de incubação ruminal.

O desaparecimento da MS diferiu significativamente $(p<0,05)$ quando as médias foram comparadas pelo teste de Tuckey, em cada um dos tempos de incubação ruminal (6h. 12h, 24h, 48h, 72h e 96h).

A grande variação no porcentual de desaparecimento da MS do BCA não tratado, fornecida pelos diversos autores (Orskov et al. ${ }^{23}, 1980$; Kumar et al. ${ }^{44}$, 1982; Vitti et al. ${ }^{30}$, 1985; Martin 18, 1988; Castro; Machado5. 1989), sugere que os resultados dependem muito da metodologia utilizada na técnica "in situ", conforme Orskov; McDonald24 (1979); Orskov et al.23 (1980); Meyer; Mackie20 (1986) e Marinucci et al. ${ }^{7}$ (1992).

Além da observação de diferenças quanto à concentração do álcali, os métodos de aplicação do $\mathrm{NaOH}$ (pulverização, ensilagem e imersão) também podem influir nos resultados (Wanapat et al. ${ }^{31}$, 1986). No presente trabalho observou-se acréscimo no desaparecimento da MS no tratamento A de 50,88 unidades (48 horas de incubação) em relação ao controle, enquanto Orskov et al.2.3 (1980) (48 horas de incubação) e Martin ${ }^{18}$ (1988) (tempo não especificado) observaram acréscimos de 40.0 (8\% de $\mathrm{NaOH}$ ) e $67,8(14 \%$ $\mathrm{NaOH})$ unidades, respectivamente.

$O$ incremento da degradabilidade no tratamento $B$, de 9,94 pontos em relação ao controle, foi relativamente pequeno, quando comparado ao obido com o tratamento por $\mathrm{NaOH}(50,88)$. Em relação aos dados observados por Villi ef al. ${ }^{30}$ ( 1985$)$, este incremento foi semelhante ao BCA tratado por pressão de vapor de $7 \mathrm{~kg} / \mathrm{cm}^{2}(10,34)$ e ao tratado biologicamente $(12,87)$.

Comparando com os valores encontrados por Martin ${ }^{18}$ (1988) e Vitti et al.30 (1985), o incremento aqui ocorrido na degradabilidade, após o tratamento por cinzas de madeira, foi inferior aos tratamentos por pressão de vapor de 14 $(22,39)$ e de $21 \mathrm{~kg} / \mathrm{cm}^{2}(21,22)$, à ensilalgem (14,43), alo tratamento por $\mathrm{NaOH}$ nas concentrações de $3 \%(16,7)$ e de $6 \%(37,4)$ e ao tratado por $\mathrm{CaOH}$ a $8 \%(31,3)$ e $16 \%(34,8)$. sendo, no entanto, superior ao tratado por amônia $(4,25)$, observado por Kumar et al. ${ }^{14}$ (1982).

A análise de regressão das curvas de degradação, utilizando-se o modelo proposto por Orskov; McDonald 24 (1979), apresentou os seguintes valores para "a": 24,12; 
I().45 e 6,40; para "b": 65,$28 ; 36,13 ; 36,42$; e para "c": 0,032 0,021: e 0,012, para soda, cinzas e água, respectivamente, sendo "a" a solubilidade (tempo zero), "b" a fração potencialmente degradável e "c" a taxa de degradação por hora.

Coomb et al. ${ }^{8}$ (1979) encontraram pequenas diferenças nas constantes da velocidade de degradação da palha tratada ou não com $\mathrm{NaOH}$, apesar de terem observado aumentos da degradabilidade da MS decorrentes do tratamento alcalino.

Orskov et al.23 (1980) apresentaram dados para a degradação da $\mathrm{MS}$ do BCA, encontrando a taxa de degradação de 0,(049 para o BCA não tratado e 0,(044 para o tratado com $8 \% \mathrm{NaOH}$, valores bem diferentes dos registrados no presente caso.

A Tab. 4 apresenta os resultados do desaparecimento de FDN $\left(65^{\circ} \mathrm{C}\right)$ do BCA tratado pelas três soluções, nos diferentes tempos de incubação ruminal.

Foram encontradas diferenças $(p<0,05)$ entre o tratamento A e os demais, pelo teste de Tuckey, em todos os tempos de incubação. O tratamento $B$, em relação ao controle, mostrou diferenças $(p<0.05)$ nos tempos de 72 e 96 horas. Os incrementos no desaparecimento da FDN em unidades porcentuais, relativamente ao controle, para 48 horas de incubação ruminal foram: $38,29 \%$ para $\mathrm{NaOH}$ e $5,89 \%$ para cinzas.

Os resultados obtidos na degradação da FDN para as frações a, b e c, por meio da regressão de Orskov; McDonald 24 (1979), foram $a=19,48,14,47$ e 14,06; b = 71,54; 36,50 e 31,$78 ; c=0,029 ; 0,024$ e 0,021 , respectivamente para soda, cinzass e água.

Coomb el al. ${ }^{8}$ (1979); Parra; Escobar ${ }^{20}$ (1985) e Canale et al.34 (1988, 1990) não observaram diferenças significativas na velocidade de desaparecimento da FDN, fato semelhante ao encontrado no presente trabalho.

As revoluções por hora ("turn-ov'er" ruminal) foram 0,07; 0,06 e 0,06 e os volumes da fase líquida ruminal $90,5 \mathrm{I} ; 84,1$ I e 91,41 I, respectivamente para soda, cinzas e água.
Os valores encontrados por Casiro; Machado 5 (1989), para rações com BCA "in natura" e hidrolisado, foram de 0,059 e 0,0827 revoluções por hora, enquanto neste trabalho foram obtidos 0,07; 0,06 e 0,06 para os tratamentos por soda, cinzas e água, respectivamente.

\section{CONCLUSÖES}

Nas condições do presente trabalho, pode-se concluir que:

1) Os tratamentos com soda e cinzas de madeira afetaram a composição do bagaço, principalmente aumentando sua fração mineral e diminuindo a fração fïbra detergente neutro. A soda exerceu maior influência do que as cinzas.

2) Em todos os tempos de incubação ruminal avaliados, a degradabilidade da matéria seca do bagaço de cana foi significativamente influenciada pelos tratamentos alcalinos por soda e cinzas. A soda exerceu maior influência do que as cinzas.

3) Para a fração fibra detergente neutro, a clegradabilidade do bagaço tratado por soda foi superior à dos tratamentos por cinzas e por água, em todos os tempos de incubação ruminal avaliados. Já a degradabilidade no tratamento por cinzas foi maior do que no tratamento por água somente nos tempos de incubação de 72 e 96 horas.

4) O tratamento do bagaço de cana por soda foi mais eficiente que por cinzas de madeira ou por água, considerando o aproveitamento do subproduto como alimento para ruminantes.

\section{AGRADECIMENTOS}

Os autores expressam seus agradecimentos aos senhores: João José Assumpção de Abreu Demarchi (Instituto de Zootecnia - Nova Odessa - SP) e José Aparecido da Cunha (FZEA-USP - Pirassununga - SP).

\section{SUMMARY}

Sugar cane bagasse was treated by immersion in one of these solutions: A) $\mathrm{NaOH} 2 \%$ volume/weigth; B) wood ash solution 30\% volume/weight and C) water alone. Treated bagasse made up $60 \%$ of dry matter ration, and the remaining $40 \%$ constituted of concentrate (corn, full fat soybean and cotton meal). Six fistulated steers in a change-over design $3 \times 3$ were used, for evaluation of fiber degradability in the rumen, through the "in situ" nylon bag technique. Dry matter degradability at $48 \mathrm{~h}$ incubation time was: $\mathrm{A}=75.1 \% ; \mathrm{B}=34.2 \%$ and $\mathrm{C}=24.2 \%$; $\mathrm{NDF}$ degradability of $\mathrm{NaOH}$ treated bagasse (A) was higher than in B or C treatments; degradability of $\mathrm{B}$ was higher than in $\mathrm{C}$ at $72 \mathrm{~h}$ and $96 \mathrm{~h}$ of incubation time. NDF degradability at 48 hour incubation time showed the following results: $\mathrm{A}=74.0 \% ; \mathrm{B}=41.6 \%$ and $\mathrm{C}=35.7 \%$. In conclusion, alkaline ( $\mathrm{NaOH}$ and wood ash) treated sugar cane bagasse improves ruminal degradability, and $\mathrm{NaOH}$ is better than wood ash.

UNITERMS: Digestibility; Cattle; Sugar cane. 


\section{REFERÊNCIAS BIBLIOGRÁFICAS}

1-ADEBOWALE, E.A. Organic waste ash as possible source of alkali for animal feed treatment. Animal Feed Science and Technology, v. 13. p.237-48, 198.5

2-CABELlO, A.; CONDE, J.; OTERO, M.A. Prediction ol the degradability of sugal cane cellulosic residues by indirect methods. Biotechnology and Bioengineering, v.23, p.2737-45, 1981

3-CANALE, C.J.: ABRAMS, S.M.: MULLER, L.D.: KJELGAARD, W. ANDERSON. P.M.: IIARPSTER. H.W. Alkali treated forage for eartly lactation dairy cows: ellect on lactation perlormance and nutrient digestibility. Journal of' Iairy Science, v.71, p.2166-74, 1988.

4-CANAI.E, C.J.: ABRAMS, S.M.: VARGA. G.A.: MULLER, L.D. Alkatreated orchardgrass and allalla: composition and "in silu" digestion of dry matter and cell wall components. Journal of Jairy Science, v.73, n.9. p. 2404-12, 1990).

5-CASTRO, I B.: MACHADO, P.F. Avaliação do processo de digestão do bagaco de cana-de-acúcar tratado sob pressão e vapor. Boletion de Indústria Animal, v.46, 11.2, p.213-7, 1989.

6-CIICCO, C.F.: GARCIA, G.: FERNANDEZ, C.: PRAYS, C.R. Cane molasses and $\mathrm{NaOH}$-treated bagacillo diets for lambs. South $\mathrm{A}$ frican Journal Animal Science, v. 13, n. I, p.18-20, 1983.

7-COCHRAN, W.G.; COX. G.M. Experimental designs. New York, John Wiley \& Sons Inc. 1957. p.611.

8-COOMB. J.B.: DINIUS, D.A. WHEELER, W.E. Elfect of alkali trealment on intake and digestion of barley straw by beef steers. Journal of Animal Science, v.49. n. I, p.169-76, 1979

9-FREITAS, E.A.G, : PATRIKA, G.F. Uso da cinza de madeira e da uréia na elevação da digestibilidade de palhas. In: REUNIÃO ANUAL DA SOCIEDADE BRASILEIRA DE ZOOTECNIA, 24, Porto Alegre, 1989. Anais, p. 150.

10-HYDEN, S. A turbidometric method for the determination of higher polyetylene glycol in biological material. Lantbru kshögskolans Annaler, v.22, p.139-45, 1986.

11-IBRAHIN M.N.M.: PEARCE, G.R. Effects of chemical pretreatment on the composition and "in vitro" digestibility of crop by-products. Agricultural Wastes, v.5, n.3, p.135-56, 1983

12-JACKSON, M.G. Review article: the alkali treatment of straws. Animal Feed Science and Technology, v.2, p, 105-30, 1977

13-JAYASURYIA, M.C.N. Potential for better utilization of crop residues and agro-industrial by-products in animal feeding in the sub-continent. FAO Animal Production and Health Paper, Rome, n.50, p.37-49, 1985.

14-KUMAR, N.: VERMA. D.N.: DASS, R.S. e SINGH, U.B. Effect of concentration and period of ammonia treatment on the chemical composition and nutritive values of sugar cane bagasse. Indian Journal Nutritional Dietetic, v. 19, p.381-8, 1982.

15-LAMAS, G.L.; SHIMADA, A.S.; RUELAS, S.C., ZUNIGA. H.M. Estudio del vilor alimenticio de subproductos de la cana de azúcal con bovinos en corral. 'Técnica Pecuaria México, v. 36, p.59-64, 1979.

16-I.AVEZZOO. W.: SILVEIRA. A.C.: MENDES. O.E.N.: CORTADA. C.N. Bagaço de callit, resíduo da agricultura tropical, como componeme de ração para ovinos. Efeitos de diferentes tratitmentos sobre os coeficienles de digestibilidade e nutrientes digestíveis totais. In: Anais do Congresso de Zootecnia do Listado de Sáo Paulo, Bolucitu, 1983. v.3, p. I I4-24.

17-MARINUCCI, M.'T'; DEHORITY, B.A.; LOERCH, S.C. In vitro and in vivo studies of lactors affecting digestion of feeds in symthetic fïher hagas. Journal of Animal Science, v. 70, p.296-307, 1992

18-MARTIN. P.C. Sugar cane as feed: bagasse lor ruminant leeding. FAO Animal Production and Healih Paper, Rome, v.72, p.134-45, 1988

19-MELOTTI, L.; I.UCCI. C.S.; NOGUEIRA FIL.HO. J.C.M.; LIMA, C.G.; l.IMA, F.R.; CUNHA, J.A. Degradabilidade ruminal de forragens nas lormas verde e desidratada. III. Degradabilidade Ruminal do Capim Napier (Pommisetum purpurem schum) em quatro lases de crescimento pela lécrica dos sacos de náilon "in situ" com bovinos fistulados. Brazilian .Journal of Veterinary Research and Animal Science, São Paulo, v 31, n. I, p.59-67, 1994.
20-MEYER, J.H.F.; MACKIE, R.I. Microbiological evaluation of the intraruminal in sacculus digestion technique. Applied Environmental Microbiology, v.51, n.3, p.622-9. 1986.

21-MOLINA, E.: BOZA, J.; AGUILERA, J.F. Nutritive value for ruminamts of sugar cane bagasse ensiled after spray treatment with diflerent levels of $\mathrm{NaOH}$. Animal Feed Science and Technology, v.9, . .1-17, 1983.

22-NOLTE, M.E.; CLINE, I.H.; DEHORITY, B.A.; IOERCH, S.C.; PARKER, C.F. Treatment of wheal straw with alkaline solutions prepared from wood ashes to improve fiber utilization by ruminants. Journal of Animal Science, v.64, p.669-77, 1987.

23-ORSKOV. E.R: DEB HOVEL, F.D.: MOULD, F. Uso de la técnica de la bolsa de nylon para la avaliación de los alimentos. Production Animal Agricultural, v.5, p.213-33, 1980.

24-ORSKOV, E.R.; MCDONAL.D. I. The estimition of protein degradability in the rumen from incubation measurements weighed according to rate of passage. Journal of Agricultural Science, v.92, p.499-503, 1979.

25-OWEN, E.: KLOPFENSTEIN, T.; URIO, N.A. Treatment with other chemicals. In: SUDSTOL, F; OWEN. E. Straw and other fibrous byproducts as feed. Amsterdam, Elsevier Science Puhlishers, 1984. p.248-73

26-PARRA, R, ESCOBAR, A. Use of Jjbrous agriculturil residues (FAR) in ruminant feeding in Latin America. FAO Animal Production and Health Paper, Rome, 11.50, p.81-98, 1985

27-RAMOREZ, R.G.; GARZA, J.; MARTONEZ, J.; AYALA, N. Wood alsh sodium hydroxide and urine to increase sorghum straw utilizalion by sheep. Small Ruminant Research, v.5, p.83-92, 1991 .

28-REDDY. M.R.; MOHAN, K.; DAS, C.T. Effect of feeding urea-molasses enriched sugar cane bagasse with or without alkali treatment as the sole source of roughage for growing cross-bred calves. Indian .Iournal Animal Research, v.15, n.1, p.9-14, 1981

29-SUDANA. I.B. The effect of fire-ash treatment on the nutritional quality of rice straw. Nutrition Abstract and Reviews, B, v.60, p. $113,1990$.

30-VITTI, D.M.S.S.; ABDALLA, A.L.; LOBÃO, A.O.; SILVA FILHO, J.C Tratamento químico, físico e biológico do bagaço de cana-de-açúcar. Comunicaçóes Científicas da Faculdade de Medicina Veterinária e Zootecnia da Universidade de São Paulo, v.9. n.2, p.139-42, 1985.

31-WANAPAT, M.: SUNDSTOL, F.; HALL. J.M.R. A comparision of alkall treatment methods used to improve the value of straw. II. In sacco and in vitro degradation relative to in vivo digestibility. Animal Feed Science and Technology, v. 14, p.215-20, 1986. 